\title{
Práticas e saberes do acolhimento na atenção primária à saúde: uma revisão integrativa
}

\author{
Pratices and knowledge of the reception in primary health care: an integrative review \\ Prácticas y conocimiento de acogida en atención primaria de salud: una revisión \\ integradora
}

Maria Vanyelle Nogueira Feitosa ${ }^{1 *}$, Raimunda das Candeias ${ }^{2}$, Anna Karuza Nogueira Feitosa ${ }^{3}$, Wesley Soares de Melo ${ }^{4}$, Flaviane Melo Araújo ${ }^{3}$, Janina Falcão do Carmo ${ }^{6}$, Francisco Daniel Rodrigues da Silva², Maria Emanuelly Crisostomo Barroso ${ }^{7}$, Mara Cibelly da Silva Pinheiro ${ }^{7}$, Aline Oliveira Rocha Brito ${ }^{7}$.

\section{RESUMO}

Objetivo: Identificar na literatura estudos que elucidem os saberes e práticas sobre acolhimento e como eles repercutem na prestação da assistência mais efetiva e humanizada. Métodos: Trata-se de uma revisão integrativa realizada no mês de maio de 2020 nas bases de dados da LILACS e BDENF. Utilizou-se Descritores em Ciências da Saúde (DeCS): acolhimento, atenção primária à saúde, estratégia de saúde da família e humanização da assistência, combinando-os com a utilização do operador booleano "AND". Elegeuse como critérios de inclusão: artigos originais com assunto principal acolhimento e humanização na APS/ESF, disponíveis na íntegra, no idioma português, inglês e espanhol e publicados nos últimos 5 anos (2014-2019). Resultados: Seis estudos foram selecionados. A apreciação dos artigos possibilitou o agrupamento em duas categorias: Acolhimento sob a ótica dos usuários e fatores que interferem no acolhimento. Considerações finais: $\mathrm{O}$ acolhimento configura-se como um grande desafio na estruturação do cuidado integral e como instrumento de importância fundamental para gestão e apreciação dos serviços de saúde.

Palavras-chave: Acolhimento, Atenção primária à saúde, Humanização da assistência.

\begin{abstract}
Objective: Identify in the literature studies that elucidate the knowledge and practices about reception and how they impact on the provision of the most effective and humanized assistance Methods: This is an integrative review carried out in May 2010 in the databases of LILACS and BDENF. Health Sciences Descriptors (DeCS) were used: reception, primary health care, family health strategy and humanization of care, combining them with the use of the Boolean operator "AND". The following inclusion criteria were chosen: original articles with the main subject of reception and humanization in the PHC / ESF, available in full, in Portuguese, English and Spanish and published in the last 5 years (2014-2019). Results: Six studies were selected. The appreciation of the articles made it possible to group them into two categories: Reception from the perspective of users and factors that interfere with reception. Final considerations: Reception is a major challenge in structuring comprehensive care and as an instrument of fundamental importance for the management and appreciation of health services.
\end{abstract}

Keywords: Reception, Primary health care, Humanization of assistance.

\footnotetext{
${ }^{1}$ Escola de Saúde Pública do Ceará (ESP-CE), Fortaleza - CE. *E-mail: vanyellefeitosa@outlook.com

2 Universidade Federal do Ceará (UFC), Fortaleza - CE.

3 Universidade Estadual Vale do Acaraú (UVA), Juazeiro do Norte - CE.

${ }^{4}$ Universidade da Integração Internacional da Lusofonia Afro-Brasileira (UNILAB), Redenção - CE.

${ }^{5}$ Universidade de Fortaleza (UNIFOR), Fortaleza - CE.

6 Universidade Estadual do Ceará (UECE), Fortaleza - CE.

${ }^{7}$ Hospital Sírio Libanês (HSL), Juazeiro do Norte e Quixadá - CE.
} 


\section{RESUMEN}

Objetivo: Identificar en la literatura reciente estudios que aclaren el conocimiento y las prácticas sobre acogida y cómo impactan en la provisión de la asistencia más efectiva y humanizada. Métodos: Esta es una revisión integradora realizada en mayo de 2010 en las bases de datos de LILACS y BDENF, utilizando los Descriptores de Ciencias de la Salud (DeCS): recepción, atención primaria de salud, estrategia de salud familiar y humanización de la asistencia, combinándolos con el uso del operador booleano "AND". Se eligieron los siguientes criterios de inclusión: artículos originales con el tema principal de recepción y humanización en el APS / FSE, disponibles en su totalidad, en portugués, inglés y español y publicados en los últimos 5 años (2014-2019). Resultados: Se seleccionaron seis estudios. La apreciación de los artículos permitió agruparlos en dos categorías: recepción desde la perspectiva de los usuarios y factores que interfieren con la recepción. Consideraciones finales: La recepción es un desafío importante en la estructuración de la atención integral y como un instrumento de fundamental importancia para la gestión y apreciación de los servicios de salud.

Palabras clave: Recepción, Atención primaria de salud, Humanización de la asistencia.

\section{INTRODUÇÃO}

O acolhimento como tecnologia leve, é uma ferramenta de cuidado que tem cooperado para amplificação do acesso aos serviços da Atenção Primária à Saúde (APS) e demais níveis do sistema de saúde, organizando o processo de cuidado do mesmo modo que melhora a oferta dos atendimentos em consonância com a demanda dos usuários (FARIAS DC, et al., 2015).

Merhy EE e Franco TB (2003) evidenciam a importância do trabalho vivo, baseado nas tecnologias leves, relacionais, para a produção de saúde. O trabalho vivo em ato, entendido pela relação entre equipe de saúde e usuário, gera colaboração mútua para a produção da saúde, reconhecendo a corresponsabilização no cuidar, tornando os sujeitos protagonistas na melhoria da qualidade da saúde, a partir da humanização da assistência, o estabelecimento de vínculo e responsabilização sanitária.

A humanização compõe um dos pilares do atual sistema de saúde vigente no Brasil, estruturado com ênfase na APS, que por sua vez, traz a Estratégia Saúde da Família (ESF), como principal porta de entrada ao sistema, ordenadora do cuidado, cujo objetivo é estabelecer vínculo entre os profissionais-usuários-família mediante uma responsabilização de toda equipe numa atenção integral capaz de melhorar fatores relacionados a saúde do usuário individual e da coletividade. A Política Nacional de Humanização (PNH) vem estabelecer que o acolhimento é uma das ferramentas características das práticas de produção e promoção da saúde (BRASIL, 2010).

A ESF se destaca pela grande contiguidade ao cotidiano da vida das pessoas e coletivo em seus territórios. Por possuir um maior grau de descentralização e capilaridade, suas equipes têm a oportunidade de se vincular e se responsabilizar, atuar na idealização de ações coletivas de promoção e prevenção no território, assim como administração dos projetos terapêuticos singulares dos usuários, transcorrendo por vezes, por outras formas de serviço, para atendimento integral das necessidades do usuário (BRASIL, 2011).

Enquanto porta de entrada preferencial do sistema único de saúde, a ESF deve se organizar para assumir sua principal função de acolher, escutar e oferecer um retorno positivo aos usuários e familiares. Deve procurar dar resolutividade as queixas de saúde e/ou minorar quaisquer tipos de prejuízos e sofrimentos retratados, ou ainda atribuir-se pela resposta a assistência ofertada, mesmo que esta seja realizada em outro serviço de atenção da rede de saúde (BRASIL, 2012).

Acolhimento não é uma atividade específica de determinada profissão, e sim uma atividade em que todos os profissionais devem estar comprometidos e capacitados para acolher os usuários, haja vista ser um instrumento de trabalho que oportuniza a produção do cuidado diferenciado, valorizando o protagonismo e a autonomia dos sujeitos bem como a corresponsabilidade entre eles (FERREIRA MLSM, et al., 2014).

A aplicabilidade do acolhimento no cotidiano das instituições de saúde funciona como uma base sólida para humanização da assistência, pois proporciona resolutividade, vínculo e responsabilização entre o profissional encarregado pelo cuidar e o usuário, o que tem cooperado na humanização da assistência e 
reorganização da atenção à saúde na ESF (ARANHA JS, et al., 2011). Assim, em virtude de sua importância como pratica de humanização, as falhas na sua implementação podem gerar inúmeros problemas de acesso aos serviços de saúde, especialmente os da Atenção Primária.

Ao se refletir sobre as mudanças positivas que o acolhimento proporciona em prol de um serviço mais organizado e uma assistência integral e humanizada ao usuário, julgou-se indispensável investigar a conjuntura do desenvolvimento de práticas e saberes acerca do acolhimento, com o propósito de colaborar com as ações de profissionais de saúde inseridos nos inúmeros serviços assistenciais da atenção primária. Nessa perspectiva, objetiva-se no presente estudo identificar na literatura estudos que elucidem os saberes e práticas sobre acolhimento e como eles repercutem na prestação da assistência mais efetiva e humanizada.

\section{MÉTODOS}

O estudo trata-se de uma revisão integrativa da literatura, a qual é um método que proporciona a síntese de conhecimento já produzido e a incorporação dos resultados desses conhecimentos na prática (MENDES KDS, et al., 2008).

Para sua elaboração, seguiu-se algumas etapas preconizadas da prática baseada em evidências: (1) identificação do tema e questão norteadora; (2) busca na literatura; (3) coleta de dados; (4) avaliação dos estudos incluídos na revisão; (5) interpretação dos resultados e; (6) apresentação da revisão integrativa (SOUZA MT, et al., 2010).

Inicialmente estabeleceu-se a seguinte questão norteadora: Os usuários e profissionais da APS/ESF sabem o que é o acolhimento? Como esse acolhimento é realizado? Como isso pode repercutir na prestação da assistência humanizada?

Para escolha das referências a serem inseridas na revisão, elegeu-se como critérios de inclusão: artigos originais contendo como assunto principal o acolhimento e humanização na APS/ESF, disponíveis na íntegra, no idioma português, inglês e espanhol e publicados nos últimos 5 anos (2014-2019). Foram excluídos estudos de caso, revisões integrativas e sistemáticas, relatos de experiência, teses, dissertações e monografias, publicações duplicadas, bem como estudos que não abordassem a temática relevante ao objetivo do estudo.

A busca das referências se deu no banco de dados da Biblioteca Virtual em saúde (BVS) durante o mês de maio de 2020, contemplando as bases de dados LILACS (Literatura Latino-Americano e do Caribe em Ciências da Saúde) e BDENF (Base de dados em Enfermagem), utilizando os descritores em ciências da saúde (DeCS): "Acolhimento"; Atenção primária à saúde"; "Estratégia de saúde da família" e "Humanização da assistência".

Coma utilização do operador booleano "AND", os descritores foram cruzados no banco de dados, obtendo incialmente 79 publicações cruzando os descritores "Acolhimento ", "Atenção primária à saúde" e "Humanização da assistência", e 60 publicações cruzando "Acolhimento", "Estratégia saúde da família" e "Humanização da assistência", totalizando 139 publicações. Essas 139 publicações foram avaliadas de acordo com os critérios de seleção anteriormente elencados, restando ao final 6 artigos que foram selecionados para leitura, explanação e análise do conteúdo.

Ainda se utilizou um instrumento previamente estruturado com finalidade de extrair as informações consideradas relevantes desses estudos, possibilitando uma melhor avaliação e maior precisão das características de cada um, interpretando e sintetizar seus resultados.

\section{RESULTADOS}

O Quadro 1 abaixo traz todos as evidências encontradas nos estudos selecionados a respeito do nosso objeto de estudo. Os dados sobre acolhimento nela compilados permitirá uma melhor visualização das informações extraídas de todos os estudos, possibilitando assim uma melhor visualização das informações extraídas, bem como a construção crítica e detalhada da revisão. 
Quadro 1 - Caracterização dos artigos analisados e inseridos na revisão. Fortaleza-CE, 2020.

\begin{tabular}{|c|c|c|c|c|c|}
\hline Autor/Ano & Idioma & Objetivo & Delineamento & $\begin{array}{c}\text { População e } \\
\text { amostra }\end{array}$ & Instrumento \\
\hline $\begin{array}{l}\text { BAIÃO } \\
\text { BDES, et al., } \\
2014 .\end{array}$ & Português & $\begin{array}{l}\text { Contribuir para a reflexão } \\
\text { dos profissionais do Posto } \\
\text { de Saúde Urbano } 01 \text { do } \\
\text { Riacho Fundo II / DF, } \\
\text { acerca do acolhimento } \\
\text { humanizado. }\end{array}$ & $\begin{array}{c}\text { Descritivo-explora- } \\
\text { tória, observacional } \\
\text { e transversal, com } \\
\text { abordagem } \\
\text { qualitativa. }\end{array}$ & $\begin{array}{l}20 \\
\text { profissionais e } \\
30 \text { pacientes. }\end{array}$ & $\begin{array}{c}\text { Entrevista } \\
\text { semiestruturada. }\end{array}$ \\
\hline $\begin{array}{l}\text { GOMES GP, } \\
\text { et al., } 2014 .\end{array}$ & Português & $\begin{array}{l}\text { Analisar o acolhimento na } \\
\text { atenção básica à saúde do } \\
\text { Riacho Fundo II. }\end{array}$ & $\begin{array}{c}\text { Descritivo-analítico, } \\
\text { quanti-qualitativo. }\end{array}$ & $\begin{array}{l}16 \\
\text { profissionais } \\
\text { de quatro } \\
\text { equipes e } 100 \\
\text { usuários. }\end{array}$ & $\begin{array}{l}\text { Observações } \\
\text { (20h), roteiro } \\
\text { estruturado e } \\
\text { técnica de grupo } \\
\text { Focal. }\end{array}$ \\
\hline $\begin{array}{l}\text { SENA ALC, } \\
\text { et al., } 2015 .\end{array}$ & Português & $\begin{array}{l}\text { Analisar a satisfação dos } \\
\text { usuários de uma Unidade } \\
\text { de Saúde da Família após a } \\
\text { implantação } \\
\text { acolhimento. }\end{array}$ & $\begin{array}{l}\text { Descritivo e } \\
\text { exploratório, } \\
\text { abordagem } \\
\text { qualitativa. }\end{array}$ & 9 usuários & $\begin{array}{l}\text { Técnica de } \\
\text { grupo focal. }\end{array}$ \\
\hline $\begin{array}{l}\text { FERREIRA } \\
\text { BR, et al., } \\
2018\end{array}$ & Português & $\begin{array}{l}\text { Descrever a visão do idoso } \\
\text { sobre o acolhimento na } \\
\text { atenção básica. }\end{array}$ & $\begin{array}{l}\text { Descritivo e } \\
\text { exploratóriocom } \\
\text { abordagem } \\
\text { qualitativa. }\end{array}$ & 15 idosos. & $\begin{array}{c}\text { Entrevista } \\
\text { semiestruturada }\end{array}$ \\
\hline $\begin{array}{l}\text { ROCHA } \\
\text { MGL, et al., } \\
2018 .\end{array}$ & Português & $\begin{array}{l}\text { Descrever as percepções } \\
\text { de mulheres atendidas em } \\
\text { uma ESF acerca do } \\
\text { acolhimento nas consultas } \\
\text { ginecológicas de } \\
\text { enfermagem. }\end{array}$ & $\begin{array}{l}\text { Descritivo com } \\
\text { abordagem } \\
\text { qualitativa. }\end{array}$ & 24 mulheres. & $\begin{array}{c}\text { Entrevista } \\
\text { semiestruturada }\end{array}$ \\
\hline $\begin{array}{l}\text { MEDEIROS } \\
\text { CB, et al., } \\
2018 .\end{array}$ & Português & $\begin{array}{l}\text { Analisar a perspectiva do } \\
\text { usuário na Atenção Básica } \\
\text { sobre o acolhimento ao } \\
\text { idoso. }\end{array}$ & $\begin{array}{l}\text { Descritivo analítico } \\
\text { com abordagem } \\
\text { qualitativa. }\end{array}$ & 30 usuários & $\begin{array}{c}\text { Entrevista } \\
\text { semiestruturada }\end{array}$ \\
\hline
\end{tabular}

Fonte: Feitosa MVN, et al. 2020.

Pode-se observar que os estudos analisados apresentaram semelhanças quanto ao delineamento e instrumentos utilizados. Tendo em vista as características desses estudos, observou que os 6(100\%) encontram-se no idioma português, com predominância das publicações no ano de 2018 (3) seguido de 2014 (2) e ainda com a totalidade da população dos estudos sendo com usuários e apenas 2 (33\%) com os profissionais da saúde.

Para identificação dos saberes, prática, entendimentos e repercussões do acolhimento, verificou-se que os $6(100 \%)$ estudos utilizaram um roteiro de perguntas abertas e fechadas, com entrevista gravada (entrevista semiestruturada) e 2 (33\%) a técnica de grupo focal.

Após síntese e análise crítica dos resultados obtidos nesses estudos, pode-se evidenciar dois grupos para discussão que foram agrupados em duas categorias temáticas: acolhimento sob a ótica dos usuários e os fatores que interferem no acolhimento.

\section{Acolhimento sob a ótica dos usuários}

Para esta temática, os estudos avaliados foram $4(66 \%)$. Dentre os discursos mais abordados no texto destacam-se duas temáticas relevantes nas falas dos usuários: Conhecimento sobre o acolhimento; e percepção/satisfação/sentimento com acolhimento.

Através da análise do conhecimento pode constatar que eles estabeleceram relação do acolhimento ao bom atendimento e tratamento pelos profissionais da recepção (FERREIRA RB, et al., 2018) ou pelo profissional na consulta (ROCHA MGLR, et al., 2018), ou ainda como sendo a dispensação da medicação após atendimento (FERREIRA RB, et al., 2018).

Quanto a percepção/satisfação/sentimento com acolhimento, os discursos revelaram que os usuários o atribuíram ao cuidado, carinho e respeito dispensado pelos profissionais (MEDEIROS CB, et al., 2018), o que 
vai além do atendimento na recepção ou consultório, o que os faz sentirem mais à vontade a aberto ao profissional. Os usuários ainda demonstraram sentimentos de satisfação com o acesso ao serviço, com a resolutividade do seu problema e com reorganização do processo de trabalho (SENA ALC, et al., 2015).

\section{Fatores que interferem no acolhimento}

Esta categoria originou-se pela análise de dois (33\%) dos estudos selecionados. Dentre os discursos de profissionais e usuários do estudo, observou-se que fatores que podem interferir e repercutir de forma negativa no acolhimento são citados como a desvalorização, rotatividade e quantidade insuficiente de profissionais, assim infraestrutura inadequada, pouco treinamento destinado às equipes, carência de materiais (BAIÃO BS, et al., 2014), e o não funcionamento da referência e contra referência (GOMES GP, et al., 2014).

Os pesquisados ainda observaram a falta de conhecimento tanto por parte dos profissionais quanto dos usuários sobre acolhimento e estratégia de saúde da família (GOMES GP, et al., 2014) e muitos dos profissionais relataram sentirem-se desvalorizados, muitas vezes, pela própria comunidade e pelos meios de comunicação em massa (BAIÃO BS, et al., 2014).

\section{DISCUSSÃO}

Compreende-se que o acolhimento no cotidiano dos serviços prestadores de saúde deve ser compreendido como uma alternativa de produzir uma realidade diferente. Pode entendido de diversas formas que se desdobram, por exemplo em saber receber, se comunicar, ouvir e dar respostas apropriadas as diferentes necessidades que surgem (BARRA AR e OLIVEIRA LML, 2012). Em relação a esse aspecto, observou que boa parte dos estudos trazem os aspectos do acolhimento sob a ótica dos usuários. Dentre estes, o atendimento dispensando desde a recepção se estendendo ao consultório profissional, assim como alguma demonstração de afeto e respeito são citadas como sendo acolhimento (FERREIRA RB, et al., 2018; ROCHA MGLR, et al., 2018; FERREIRA RB, et al., 2018).

Neste quesito, pode-se constatar nos estudos que eles estabeleceram relação do acolhimento ao bom atendimento e tratamento pelos profissionais da recepção (FERREIRA RB, et al., 2018) ou pelo profissional na consulta (ROCHA MGLR, et al., 2018), ou ainda como sendo a dispensação da medicação após atendimento (FERREIRA RB, et al., 2018).

Quanto a isso, de fato, é evidente que quando desde a chegada o usuário é bem recebido/acolhido pelos profissionais da unidade, há abertura de espaços para confiança destes (OLIVEIRA LML, et al., 2008). Quando os profissionais assumem como sua responsabilidade a assistência do usuário, pautada no respeito, cuidando com um olhar holístico, buscando a valorização das pessoas envolvidas no processo de saúde e doença, há estreitamento de vínculos e fortalecimento da relação, reduzindo desta maneira diversos agravos que possam existir, principalmente, quando o vínculo se encontra fragilizado, dificultando que o cuidar aconteça de forma integral (SILVA MZN, et al., 2014).

Além do mais, compreender o acolhimento como lógica de prevenção e promoção da saúde, também é algo fundamental para o desenvolver das ações e requer dos profissionais a compreensão da necessidade do longo processo de cuidar. Para isso, ele precisa ser iniciado desde a recepção, compreendida como a porta de entrada da unidade, porém não resumindo somente a ela. Ele perpassa todo o processo da chegada até a resolução do problema, possibilitando regular o acesso desse usuário através da prestação de ações e serviços mais adequados, que contribui para a satisfação do usuário (SMSDC, 2011).

O bom acolhimento e o vínculo com profissional/equipe são imposições que seguem os sujeitos em toda rede de atenção, sendo condição para satisfação e busca pela assistência integrada. Quando traduzido em forma de acesso, o acolhimento pode reorganizar o serviço de saúde bem como qualificar a assistência ofertada (SILVA MZN, et al., 2014), dando suporte para satisfação de outras necessidades apresentadas pelo sujeito, como por exemplo o apoio familiar, que quando desenvolvido de forma satisfatória contribui para que haja a necessidade de dar continuidade ao tratamento (SILVA TF e ROMANO VF, 2015).

Sumarizando, o acolhimento possui características que promove o desenvolvimento de vínculo usuárioprofissional, viabilizando o encorajamento ao autocuidado, maior entendimento sobre sua situação de saúde 
e maior comprometimento com o tratamento implementado. $\mathrm{O}$ acolhimento ainda contribui para o acesso universal e humanização da assistência prestada, tornando mais forte o trabalho multiprofissional e intersetorial, além incentivar práticas de enfretamento a qualquer tipo de preconceito que possam surgir (GARUZI M, et al., 2014).

Nos estudos, observou-se ainda uma narrativa dos usuários sobre a percepção/satisfação/sentimento com acolhimento. Os discursos revelaram que os usuários o atribuíram ao cuidado, carinho e respeito dispensado pelos profissionais (MEDEIROS CB, et al., 2018). Os usuários ainda demonstraram sentimentos de satisfação com o acesso ao serviço, com a resolutividade do seu problema e com reorganização do processo de trabalho (SENA ALC, et al., 2015).

No tocante a esses aspectos vários fatores podem influenciar, mas a melhora em seu estado de saúde decorrente do acolhimento é de longe perceptível. Isto está relacionado não somente à resolução do seu problema de saúde, mas também à abertura de diálogo que possibilita a exposição de dúvidas, insegurança, medo, aflição, permitindo a criação de novos caminhos e oportunidade de inter-relação no processo de saúdedoença (MACEDO CA, et al., 2011; MEDEIROS CB, et al., 2018).

Mesmo marcado por muitas dificuldades para a sua aplicação, o acolhimento corrobora para organizar a dinâmica do trabalho e melhorar a demanda, citadas como pontos satisfatórios pelos usuários dos estudos elencados na revisão (SENA ALC, et al., 2015). Isto porque além desta organização do processo de trabalho que reflete na resolutividade, o acolhimento também amplia a visão da equipe para as necessidades dos usuários, que vai além da identificação nosológica, sendo atitudes que perpassam as possibilidades dos serviços prestadores de assistência à saúde (SILVA TF e ROMANO VF, 2015). Fala-se de abandonar uma abordagem meramente mecanizada e mudar o olhar para o subjetivo, sem ancorar-se somente nos sinais e sintomas, que muitas vezes são passíveis momentaneamente de alívio por um simples gesto de respeito e paciência.

Já no que diz aos fatores que podem interferir e repercutir de forma negativa no acolhimento em uma unidade de saúde, os estudos relataram diversos fatores como a desvalorização do profissional de saúde, rotatividade e quantidade insuficiente de profissionais, infraestrutura inadequada, pouco treinamento destinado às equipes, carência de materiais (BAIÃO BS, et al., 2014), e o não funcionamento da referência e contra referência (GOMES GP, et al., 2014). Os pesquisadores ainda observaram a falta de conhecimento tanto por parte dos profissionais quanto dos usuários sobre acolhimento e estratégia de saúde da família (GOMES GP, et al., 2014), além de muitos dos profissionais participante dos estudos relataram sentirem-se desvalorizados pela própria comunidade e pelos meios de comunicação em massa (BAIÃO BS, et al., 2014).

Analisando a literatura sobre acolhimento, observa-se que há um consenso sobre os sentidos que este traz, que vão desde recepção do usuário até atenção resolutiva. No entanto, mesmo diante de um leque de conceitos e definições, todos incidem para descrições que o traz como uma diretriz do processo de trabalho dirigido ao usuário e que organiza o serviço de saúde, objetiva à garantia dos princípios do Sistema Único de Saúde e o atendimento mais humanizado (GARUZI M, et al., 2014).

Quanto a isso, é enfático os resultados nos estudos que mostram a falta de conhecimento ou concepção errôneo sobre acolhimento tanto pelos usuários quanto pelos profissionais. Isso acaba prejudicando o desenvolvimento adequado do acolhimento, prejudicando a assistência a ser ofertada e o usuário sai da unidade sem resolução do problema.

Estudo realizado com usuários do sistema de saúde aponta que os usuários sentem satisfação com o acolhimento quando não há demora no agendamento e atendimento de consultas e quando há pouca dificuldade do acesso. A humanização do cuidado também repercutiu para a qualidade da atenção à saúde, pois quando este se sentiu acolhido, foi ouvido e examinado, houve satisfação com o atendimento. Entretanto, figura do médico para os usuários ainda constitui em potência a necessidade para resolução de seus problemas (GOMIDE MFS, et al., 2018).

Em outro estudo na literatura, a partir da visão de profissionais de saúde, evidenciou-se que ainda é predominante o trabalho nas unidades de saúde nos moldes tradicionais, centrados na consulta médica, na 
distribuição de fichas para demanda espontânea, sem de fato existir a resolução do problema do usuário não contemplando sua necessidade. Por isso, a importância do acolhimento, pois pode orientar e direcionar o atendimento apropriado à demanda apontada pelo usuário (CAMELO MS, et al., 2016).

Muitos destes erros se encontram em uma concepção medicalizante da assistência e o paradigma biomédico ainda existente, configurando as necessidades dos indivíduos, o atendimento prestado pelos demais profissionais e processo de organização do sistema, podendo induzir a uma incredulidade nos resultados da atenção primária (CAMPOS RTO, et al., 2014; FERREIRA RB, et al., 2018).

Em decorrência disso, a atuação de uma equipe multiprofissional atuante na clínica solidária, que quebre com a construção de procedimentos focados em atos prescritos e na doença, possibilita moldar o avanço legal à prática diária dos serviços prestadores de saúde (SATO M e AYRES JRCM, 2015). Para ser acolhido, o atendimento não necessita decorrer de consultas médicas, mas da comunicação/diálogo ou a escuta que diversas vezes pode resolver o problema que aflige o usuário.

O conhecimento deve ser mútuo entre os sujeitos do processo, destacando o respeito aos posicionamentos individuais na construção de uma assistência integral. Neste sentido, sugere-se a fomentação de movimentos que tornem os profissionais conhecedores dessa perspectiva e tentem restabelecer, através do diálogo, o conceito de que o usuário é parte do processo e deve ser assim acolhido (STORINO LP, et al., 2013; CANDEIAS R, et al., 2019).

Já no entendimento do conceito sobre ambiência, este pode ser compreendido além do espaço que visa conforto e privacidade individual das pessoas envolvidas. Este é entendido como espaço de construção de subjetividades e como instrumento facilitador do trabalho, que propicia a melhoria de recursos, uma atenção humanizada, acolhedora e resolutiva (BAIÃO BS, et al., 2014). Profissionais norteados por tal entendimento e que o incorpora nos serviços de saúde produz cuidados mais humanizados e orienta de fato o trabalho (BARROS MMMA, et al., 2010).

Sato M e Ayres JRCM (2015) enfatizam que a utilização da artística, auxiliando-se de pinturas produzidas pelos próprios usuários e profissionais, na sala de espera viabiliza a humanização no serviço, em especial se a manifestação da arte parte dos profissionais e usuários, oportunizando a manifestação dos almejos que os levam a se fazem presentes na unidade.

Um outro problema é a falta de investimento na contratação e qualificação dos profissionais (FERREIRA MLSM, et al., 2014). Este ponto pode ser um dos reflexos da falta de participação efetiva dos gestores e 0 fomento em educação continuada. É de responsabilidade da gestão de saúde promover estímulos aos profissionais no que tange às abordagens teóricas e práticas sobre o acolhimento, visto que são subsídios primordiais na concretização de um método humano e íntegro. Isso lhes dá mais segurança no que faz e, fazendo de competente e correta gera satisfações e valorização entre os sujeitos.

No mais, o sistema de referência e contra referência quando não funciona de forma adequada, desorganiza o acesso aos outros serviços e perde a continuidade da assistência (GOMES GP, et al., 2014). O acolhimento comporta-se como a mola mestre, a qual permite a humanização nos diversos ambientes do cuidado. Ressalta-se que essa maior sensibilização pode ser experimentada através das diversas necessidades, podendo serem resolvidas na unidade ou encaminhadas para outras unidades da complexidade competente, buscando sempre a resolubilidade da demanda solicitada (SILVA TF e ROMANO VF, 2015).

Para alcance dos objetivos da humanização, a política propõe a formação de equipe multiprofissional corresponsável onde tanto o usuário quanto o profissional façam parte do processo de solução dos problemas e tenham as necessidades atendidas quando chegam a unidade. Porém, na maioria das vezes, o profissional se sente despreparado, mostrando diversas dificuldades que intervém no exercer de suas atividades junto ao usuário/população, o que acaba por comprometer a assistência (GOMES GP, et al., 2014).

Assim, o acolhimento deve ser entendido como um instrumento forte e de grande potência para dar resposta aos questionamentos que surgem nos mais diferentes ambientes de saúde. Essa ferramenta é capaz de promover acesso, proporcionar conexão entre equipe/população, trabalhador/usuário, questionar o processo de trabalho desenvolvido, estimular o cuidado pleno e transformar a clínica. Dessa forma, é 
imprescindível a qualificação de trabalhadores desde a recepção, para acolher forma mais humanizada, atender adequadamente a demanda, escutar as queixas, dialogar com equipe, amparar as dificuldades, orientar e negociar (CAMELO MS, et al., 2016).

Todos esses fatores mencionados nos estudos podem interferir direta ou indiretamente na implantação e pratica de um atendimento acolhedor e humanizado, contribuindo assim para insatisfação dos usuários e profissionais.

\section{CONSIDERAÇÕES FINAIS}

O acolhimento configura-se como um grande desafio na estruturação do cuidado integral e como instrumento de importância fundamental para gestão e apreciação dos serviços de saúde. A criação de vínculo e o acolher o usuário, são fundamentais para satisfação deste nas suas necessidades de saúde. Então, formar movimentos que tornem os profissionais conhecedores deste aspecto e de forma dialógica reconfigurar a concepção dos usuários a respeito da percepção da estratégia como um espaço de pronto atendimento e a assistência medicalizante com enfoque no profissional médico, parece ser uma saída fundamental.

\section{REFERÊNCIAS}

1. ARANHA JS, et al. Acolhimento e humanização: perspectiva do atendimento na atenção básica. Informe-se em promoção da saúde, 2011; 7(2):23-24

2. BAIÃO BS, et al. Acolhimento humanizado em um posto de saúde urbano do Distrito Federal, Brasil. Revista APS. 2014; 17(3): 291302.

3. BARRA AR, OLIVEIRA LML. O acolhimento na atenção primária à saúde: dispositivo disparador de mudanças na organização do processo de trabalho? Revista APS, 2012; 15(2):126-138.

4. BARROS MMMA, et al. Prática de saúde mental na rede de atenção psicossocial: a produção do cuidado e as tecnologias das relações no discurso do sujeito coletivo. Revista APS, 2010; 13(1):72-83.

5. BRASIL. Ministério da Saúde. Secretaria de Atenção à Saúde. Núcleo Técnico da Política Nacional de Humanização. Acolhimento nas práticas de produção de saúde. Brasília (DF): MS; 2010.

6. BRASIL. Ministério da Saúde. Secretaria de Atenção à Saúde. Acolhimento à demanda espontânea. Série A. Normas e Manuais Técnicos Cadernos de Atenção Básica, n. 28, Volume I, Brasília - DF 2011.

7. BRASIL. Ministério da Saúde. Secretaria de Atenção à Saúde. Departamento de Atenção Básica. Política Nacional de Atenção Básica / Ministério da Saúde. Secretaria de Atenção à Saúde. Brasília, DF: Ministério da Saúde, 2012.

8. CAMELO MS, et al . Acolhimento na atenção primária à saúde na ótica de enfermeiros. Acta paul. Enferm., 2016; 29(4): 463-468.

9. CAMPOS RTO, et al. Avaliação da qualidade do acesso na atenção primária de uma grande cidade brasileira na perspectiva dos usuários. Saúde Debate, 2014; 38(n. especial):252-264.

10. CANDEIAS R, et al. Acolhimento na estratégia saúde da família: concepções e práticas. Rev Norte Mineira de Enferm, 2019; 8(1):4957.

11. FARIAS DC, et al. Acolhimento e Resolubilidade das Urgências na Estratégia Saúde da Família. Rev Bras EducMéd, 2015.39(1):1981-5271.

12. FERREIRA MLSM, et al. Acolhimento na percepção dos enfermeiros da Atenção Primária à Saúde. Aquichan, 2014; 14(2): $216-225$.

13. FERREIRA RB, et al. Acolhimento ao Idoso na Atenção Básica: Visão do Usuário. Rev Fund Care Online, 2018; 10(3):669-674.

14. GARUZI M, et al. User embracement in the Family Health Strategy in Brazil: an integrative review. Rev Panam Salud Publica, 2014; 35(2):144-9.

15. GOMES GP, et al. A análise do acolhimento na perspectiva das equipes de saúde da família e dos usuários no centro de saúde da família 04 do Riacho Fundo II. Revista APS, 2014; 17(3):325-333.

16. GOMIDE MFS, et al. A satisfação do usuário com a atenção primária à saúde: uma análise do acesso e acolhimento. Interface Comunicação, Saúde, Educação, 2018; 22(65): 387-398.

17. MACEDO CA, et al. Possibilidades e limites do acolhimento na percepção de usuários. Revista Enfermagem, $2011 ; 19(3): 457-462$.

18. MEDEIROS CB, et al. A perspectiva do usuário na atenção básica sobre o acolhimento ao idoso. Revista Ciência Plural, 2018; 4(3):43-56.

19. MENDES KDS, et al. Revisão Integrativa: métodos de pesquisa para a incorporação de evidências na saúde e na enfermagem. Texto Contexto Enfermagem, 2008; 17(4):758-764.

20. MERHY EE, FRANCO TB. Por uma Composição Técnica do Trabalho Centrada nas Tecnologias Leves e no Campo Relacional in Saúde em Debate, Ano XXVII, v.27, N. 65, Rio de Janeiro, Set/Dez de 2003

21. OLIVEIRA LML, et al. Acolhimento: concepções, implicações no processo de trabalho e na atenção em saúde. Revista APS, 2008; 11(4):362-373.

22. ROCHA MGLR, et al. Acolhimento na consulta ginecológica de enfermagem: percepções de mulheres da Estratégia Saúde da Família. Revista Rene, 2018;19: e3341.

23. SATO M, AYRES JRCM. Arte e humanização das práticas de saúde em uma Unidade Básica. Comunicação Saúde Educação, 2015; 19(55):1027-1038.

24. SMSDC. Secretaria Municipal de Saúde e Defesa Civil. Superintendência de Atenção Primária. Guia de Referência Rápida. Carteira de Serviços: Relação de serviços prestados na Atenção Primária à Saúde. Rio de Janeiro: SMSDC, 2011. 128 p.

25. SENA ALC, et al. Acolhimento e satisfação do usuário na estratégia de saúde da família: uma experiência de êxito. Rev. APS, 2015; 18(2): $134-140$.

26. SILVA MZN, et al. Acesso e acolhimento no cuidado pré-natal à luz de experiências de gestantes na Atenção Básica. Saúde Debate, 2014; 38(103):805-816.

27. SILVA TF, ROMANO VF. Sobre o acolhimento: discurso e prática em Unidades Básicas de Saúde do município do Rio de Janeiro. Saúde Debate, 2015; 39(105):363-374.

28. SOUZA MT, et al. Revisão integrativa: o que é e como fazer. Einstein, 2010; 8(1):102-106.

29. STORINO LP, et al. Necessidades de saúde de homens na atenção básica: acolhimento e vínculo como potencializadores da Integralidade. Escola Ana Nery, 2013; 17(4):638-645. 